\title{
High-Contrast Switching of Plasmonic Structural Colors: Inorganic versus Organic Electrochromism
}

\author{
Marika Gugole, ${ }^{\ddagger}$ Oliver Olsson, ${ }^{\ddagger}$ Kunli Xiong, Jolie C. Blake, José Montero Amenedo, \\ Ilknur Bayrak Pehlivan, Gunnar A. Niklasson, and Andreas Dahlin*
}

Cite This: ACS Photonics 2020, 7, 1762-1772

Read Online

\section{ACCESS | Llll Metrics \& More | 回 Article Recommendations | (s) Supporting Information}

ABSTRACT: Plasmonic structural colors have recently received a lot of attention. For many applications there is a need to actively tune the colors after preparing the nanostructures, preferably with as strong changes in the optical response as possible. However, to date, there is a lack of systematic investigations on how to enhance contrast in electrically induced color modulation. In this work we implement electrochromic films with plasmonic metasurfaces and compare systematically organic and inorganic materials, with the primary aim to maximize brightness and contrast in a reflective color display. We show nanostructures with good chromaticity and high

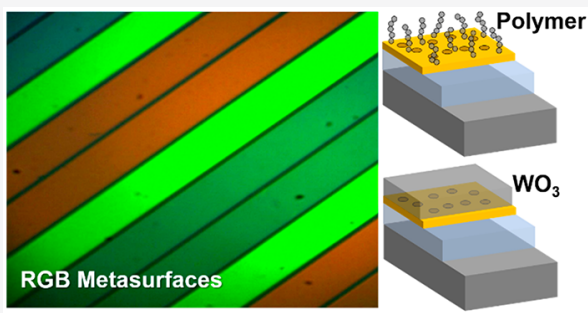
polarization-insensitive reflectivity $(\sim 90 \%)$ that are electrochemically stable in a nonaqueous solvent. Methods are evaluated for reliable and uniform electropolymerization of the conductive polymer dimethylpropylenedioxythiophene (PProDOTMe $\mathrm{C}_{2}$ ) on gold. The resulting organic films are well-described by Lambert-Beer formalism, and the highest achievable contrast is easily determined in transmission mode. The optical properties of the inorganic option $\left(\mathrm{WO}_{3}\right)$ require full Fresnel models due to thin film interference, and the film thickness must be carefully selected in order to maintain the chromaticity of the metasurfaces. Still, the optimized fully inorganic device reaches the highest contrast of approximately $60 \%$ reflectivity change for all primary colors. The switching time is about an order of magnitude faster for the organic films (hundreds of ms). The bistability is very long (hours) for the inorganic devices and comparable for the polymers, which makes the power consumption essentially zero for maintaining the same state. Finally, we show that switching of the primary colors in optimized devices (both organic and inorganic) provides almost twice as high brightness and contrast compared to existing reflective display technologies with RGB subpixels created by color filters.

KEYWORDS: metasurfaces, structural colors, electrochromism, reflective display, electronic paper

$\mathrm{P}$ lasmonic nanostructures have found a broad range of applications, as they offer control of electromagnetic fields on the nanoscale. One application with strong recent interest is structural colors arising from resonances that occur in the visible. ${ }^{1}$ Utilized since ancient times, ${ }^{2}$ such colors have advantages such as high stability compared to organic dyes and the possibility to "print" with very high resolution compared to ink. ${ }^{1}$ Also, due to the strong interaction with light, ultrathin $(<1 \mu \mathrm{m})$ nanostructures or "metasurfaces" are sufficient to generate vibrant colors. ${ }^{3}$ Recently, there has also been an increased interest in active plasmonic devices, where the optical properties can be changed on demand. ${ }^{4-8}$ In particular, tuning of structural colors in this manner is highly interesting for new types of displays. ${ }^{9,10}$ Modulation of transmitted or reflected light can be achieved in many ways. ${ }^{9}$ For instance, liquid crystals can be implemented, even for addressing individual pixels. ${ }^{11}$ Another approach is to create hybrid materials containing the plasmonic metal and conductive polymers, which makes it possible to perform redox switching in electrochemical cells. ${ }^{5,12-15}$ Alternatively, inorganic electrochromic materials have been considered for display applications since the pioneering work of Deb. ${ }^{16}$
Transition metal oxides, in particular, $\mathrm{WO}_{3},{ }^{17}$ have been frequently studied due to their application in electrochromic windows. ${ }^{18}$ Just during the last year, there has been a strong interest in implementing inorganic materials for new types of electrochromic devices. ${ }^{4,6,7}$ Both organic and inorganic electrochromic materials are convenient to implement on plasmonic nanostructures, as they often have continuous metals that act as supporting electrodes. It may also be possible to electrochemically synthesize the electrochromic layer directly on the nanostructure. ${ }^{5,13}$

However, to date, there has been relatively little work on how to optimize the optical contrast in active plasmonic devices that transmit, reflect or scatter light. This is of obvious importance for tuning structural colors. ${ }^{10}$ For plasmonic devices, early work was to a high extent focused on electrical

Received: March 11, 2020

Published: May 28, 2020 
tuning of resonance shifts rather than intensity modulation. ${ }^{14,15}$ In the field of electrochromic polymers (or their inorganic counterparts) high optical contrast, defined as difference in reflectivity or transmittance $(\Delta R$ or $\Delta T)$, has long been the central benchmark because the spectra typically do not show any strong resonance features. ${ }^{19-22}$ For plasmonic colors, high contrast is necessary if the nanostructures should be useful in reflective (or possibly "transflective") displays that rely on ambient light. Such "electronic paper" technologies are normally operating in black and white (electrophoretic displays or LCDs) due to the low reflectivity and contrast in color mode. ${ }^{10}$ For instance, liquid crystals require polarizers that naturally only transmit $50 \%$ of the incident light. An additional intensity loss comes from the fact that, for color generation, subpixels are normally created by colored glass filters, which means that only a fraction of the area will display the set color. $^{23}$ An alternative subpixel design is found in the electrowetting display based on colored liquids, ${ }^{24}$ but to date this technology has not made it to the market. In order to make electronic paper in color with good visual appearance, RGB subpixels must have both high absolute reflectivity and high switching contrast. Naturally, high intensity changes are also important for many other active plasmonic devices, such as tunable filters and so on. ${ }^{12,13}$ Looking at existing literature, it remains unexplored in what ways inorganic materials can provide better performance than conjugated polymers. At the same time, there is currently a strong interest in combining inorganic electrochromism with plasmonics, but so far it has been used for tuning cavity resonances ${ }^{4,7}$ or regulation of photothermal effects. ${ }^{2 S}$

In this work we present plasmonic metasurfaces that fulfill the main criteria for subpixels in a reflective color display: good chromaticity, high reflectivity, and, in particular, high-contrast switching. By selecting the right metals, we show that electrochromic switching is possible in nonaqueous electrolytes, which makes it possible to prepare highly stable and fully inorganic devices. Simple transmission measurements are shown to be sufficient for evaluating the maximum possible contrast of organic electrochromic films, while more advanced Fresnel models are used for the inorganic films. We show a systematic comparison of the highest achievable contrast in structural color switching using established organic and inorganic electrochromic materials. Under optimal conditions, our electrochromic metasurfaces provide sufficient brightness and contrast to clearly outperform existing reflective color display technologies that rely on glass filters to generate subpixels. In addition to contrast, we look at other parameters such as switching speed and power consumption. Although this study focuses on reflective color displays, our results should also be of general interest for other active plasmonic devices.

\section{THEORY}

We first overview the theory for evaluating switching contrast before applying it in experiments. Consider a thin absorbing film of thickness $d$. If interference effects can be ignored, the transmitted light intensity $I$ (incident intensity $I_{0}$ ) through the film follows the Lambert-Beer law: ${ }^{26}$

$$
E(\lambda)=\log \left(\frac{I_{0}(\lambda)}{I(\lambda)}\right)=\log \left(\frac{1}{T(\lambda)}\right)=\alpha(\lambda) d
$$

Here, $E$ is the extinction, $T$ is the transmittance, and $\alpha$ is the absorbing capacity of the layer (inverse length, i.e., number density multiplied by cross-section). In reflection mode, the incident light passes through the film twice and the derivation is analogous. Upon switching, $\alpha$ changes between two values representing the colored and bleached states. The change in reflectivity is ${ }^{19}$

$$
\Delta R=\left[\exp \left(-2 \alpha_{\mathrm{ble}} d\right)-\exp \left(-2 \alpha_{\mathrm{col}} d\right)\right] R_{0}
$$

Here $R_{0}(\lambda)$ is the reflectivity of the underlying surface. ${ }^{27}$ The thickness that gives highest contrast can be calculated by deriving eq $2:^{19^{8}}$

$$
d_{\mathrm{opt}}=\frac{\log \left(\frac{\alpha_{\mathrm{col}}}{\alpha_{\mathrm{ble}}}\right)}{2\left[\alpha_{\mathrm{col}}-\alpha_{\mathrm{ble}}\right]}
$$

Now we insert $d_{\text {opt }}$ into eq 2 to get the highest contrast:

$$
\begin{aligned}
& \frac{\Delta R_{\max }}{R_{0}}=\frac{\{\Delta R\}_{d=d_{\mathrm{opt}}}}{R_{0}}=\exp \left(-\alpha_{\mathrm{ble}} \times \frac{\log \left(\frac{\alpha_{\mathrm{col}}}{\alpha_{\mathrm{ble}}}\right)}{\left[\alpha_{\mathrm{col}}-\alpha_{\mathrm{ble}}\right]}\right) \\
& -\exp \left(-\alpha_{\mathrm{col}} \times \frac{\log \left(\frac{\alpha_{\mathrm{col}}}{\alpha_{\mathrm{ble}}}\right)}{\left[\alpha_{\mathrm{col}}-\alpha_{\mathrm{ble}}\right]}\right)=\left[\frac{\alpha_{\mathrm{col}}}{\alpha_{\mathrm{ble}}}\right]^{1 /\left(1-\alpha_{\mathrm{col}} / \alpha_{\mathrm{ble}}\right)} \\
& -\left[\frac{\alpha_{\mathrm{col}}}{\alpha_{\mathrm{ble}}}\right]^{\alpha_{\mathrm{col}} / \alpha_{\mathrm{ble}} /\left(1-\alpha_{\mathrm{col}} / \alpha_{\mathrm{ble}}\right)}
\end{aligned}
$$

Remarkably, this shows that the highest achievable contrast $\Delta R_{\max }$ in absolute numbers, depends only on the ratio $\alpha_{\mathrm{col}} / \alpha_{\mathrm{ble}}$ (and $R_{0}$ ). Previous studies have arrived at similar conclusions, ${ }^{20,22}$ but, as far as we are aware, eq 4 has not been presented. We emphasize that it does not contain the film thickness. There is a certain thickness that gives the highest contrast (eq 3), but there is no need to know the thickness of the analyzed film in order to obtain the ratio $\alpha_{\mathrm{col}} / \alpha_{\mathrm{ble}}$ and, hence, the highest possible contrast. The critical ratio can be measured very easily because, by definition (eq 1), it is equal to the extinction ratio:

$$
\frac{\alpha_{\mathrm{col}}}{\alpha_{\mathrm{ble}}}=\frac{E_{\mathrm{col}}}{E_{\mathrm{ble}}}
$$

Hence, the highest contrast can be determined directly by the ratio of extinction values for the colored and bleached states using transmission mode measurements, as long as the background extinction is properly subtracted. Note that the thickness may change between colored and bleached states for organic films, ${ }^{28}$ but this only introduces a dimensionless "swelling factor" into the $\alpha$ values, and the end result is the same. Note also that the highest contrast in transmission mode is identical $\left(\Delta T_{\max }=\Delta R_{\max }\right)$ according to this model, but it occurs for a twice as thick film.

To get an accurate opaque/transparent type of contrast value across the visible, which accounts for the variation of eye receptor sensitivity with wavelength, the luminosity function can be used as weight:

$$
\overline{\Delta R}=\frac{\int_{380 \mathrm{~nm}}^{780 \mathrm{~nm}} L(\lambda) \Delta R(\lambda) \mathrm{d} \lambda}{\int_{380 \mathrm{~nm}}^{780 \mathrm{~nm}} L(\lambda) \mathrm{d} \lambda}
$$

Like in our initial work, ${ }^{3}$ we use the $L(\lambda)$ function representing photoptic vision (good illumination conditions) since this is the likely scenario for display of structural colors. 


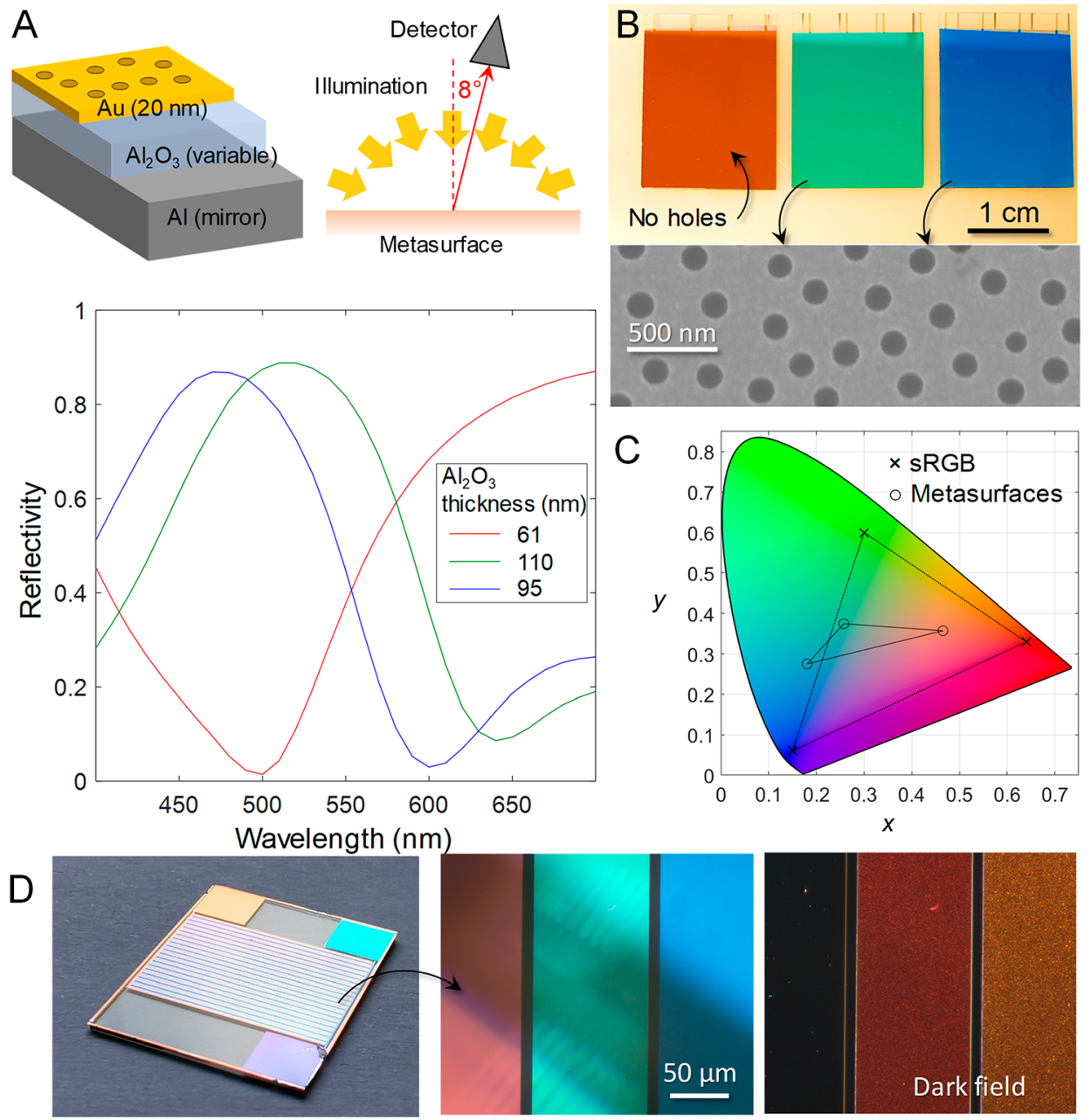

Figure 1. Metasurfaces based on an $\mathrm{Al}$ mirror, variable $\mathrm{Al}_{2} \mathrm{O}_{3}$ thickness, and a semitransparent Au with nanoholes. (A) Reflectivity in air under diffuse illumination (simulating ambient light) and collection angle of $8^{\circ}$. The values for alumina thickness represent the optimal primary colors. (B) Photo of metasurfaces. The electron microscopy image shows the nanoholes (not present on red samples). (C) CIE chromaticity coordinates compared to the standard RGB gamut. (D) A "white" surface made by RGB stripes. The microscopy images show the colors in bright or dark field illumination (same position).

To account for thin film interference, more advanced Fresnel models (transfer matrix method) can be used. ${ }^{29}$ This requires accurate determination of the complex and wavelength-dependent permittivity of the electrochromic material in different oxidation states, which is fairly complicated. ${ }^{30}$ The method based on the extinction ratio has the advantage that it only requires simple transmission mode spectra. These theoretical descriptions serve as guidelines when optimizing contrast (as shown below). In order to account for the plasmonic field enhancement, numerical simulations are preferable since the near-field distribution is multidimensional in nanostructures. ${ }^{3}$

\section{RESULTS AND DISCUSSION}

Structural color generation was achieved by a combination of Fabry-Pérot modes (cavity resonances) and plasmonic effects using a triple layer consisting of a metal mirror, an insulator with varied thickness, and a thin top metal film with nanoholes. ${ }^{3,27}$ The structures were prepared easily over large areas by colloidal lithography. Several different metals were tested, but not all were sufficiently stable in electrochemical cells, even with protective coatings. ${ }^{27}$ In particular, the electrolyte imposes limitations as metals can dissolve in some solvents or react with certain ions. ${ }^{31} \mathrm{We}$ found that a combination of an aluminum base mirror and gold as the top thin film provided structures with good color quality and stability over a broad voltage range. For instance, it was possible to use nonvolatile and inert electrolytes such as propylene carbonate with $\mathrm{LiClO}_{4} \cdot{ }^{17}$ Nonaqueous electrolytes are advantageous for device stability since unwanted Faradaic processes such as hydrolysis and gas formation can be avoided. It was also possible to directly dissolve the monomers for electropolymerization, which would require surfactants in aqueous environments. ${ }^{3}$

In order to evaluate the metasurface colors under ambient light, the reflectivity was measured with diffuse illumination using an integrating sphere (Figure 1A). This is a standard colorimetry test (ISO 17025, used, for instance, in the textile industry) that provides an accurate representation of ambient illumination in most scenarios. Thus, we consider the results in Figure 1 to be an accurate representation of the colors, even though fixed angle (specular) reflection could lead to better chromaticity. The optimal primary colors (R, G, B) were achieved with 61,110 , and $95 \mathrm{~nm}$ of $\mathrm{Al}_{2} \mathrm{O}_{3}$ spacer thickness. Photos and an electron microscopy image of RGB metasurfaces are shown in Figure $1 \mathrm{~B}$. Note that there was 

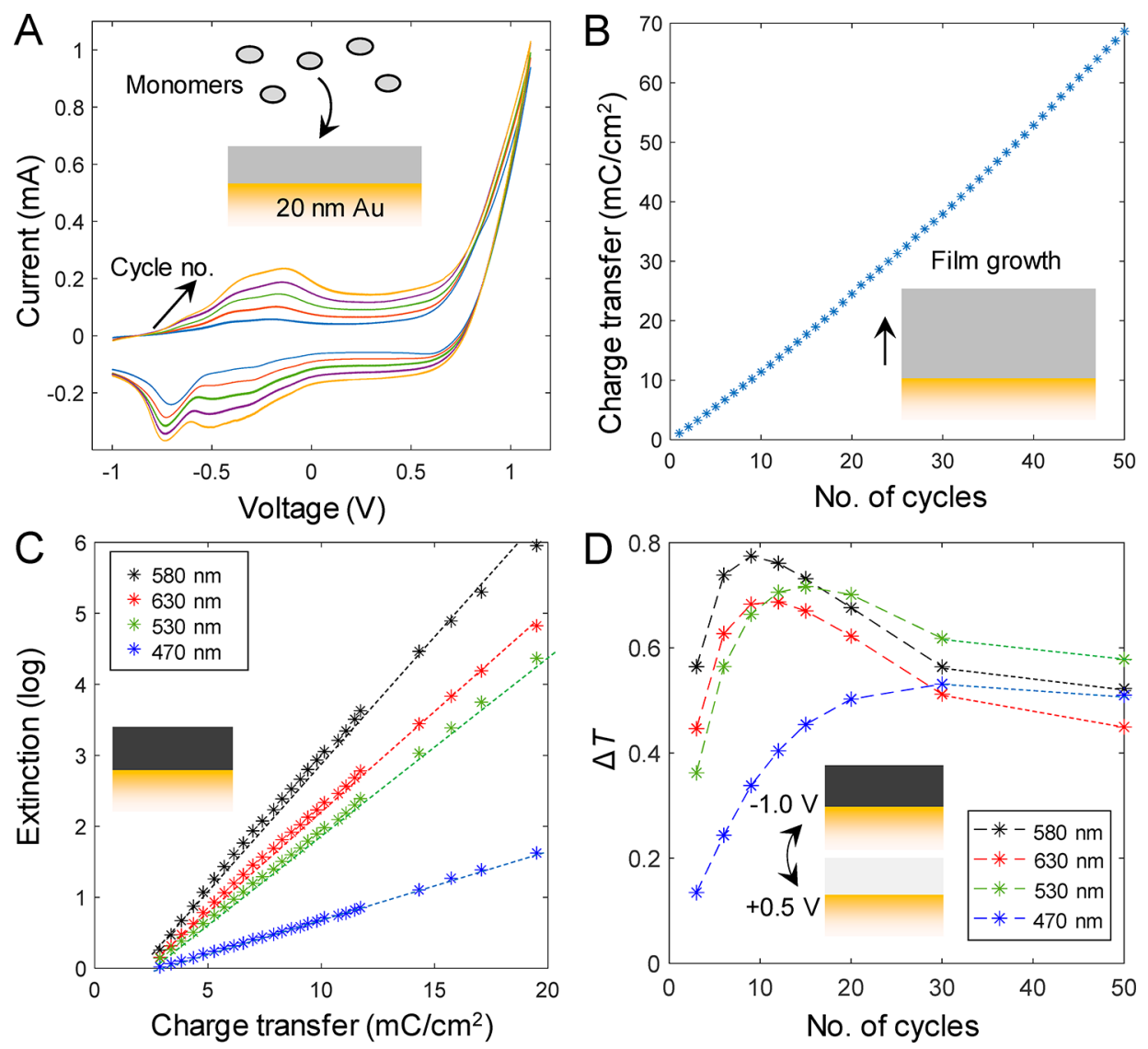

Figure 2. Electropolymerization and optical characterization of PProDOTMe $\mathrm{P}_{2}$ on $\mathrm{Au}$. (A) Current during the first $5 \mathrm{CV}$ scans for electropolymerization, represented by the current increase at $+0.8 \mathrm{~V}$. The scan rate was $200 \mathrm{mV} / \mathrm{s}$ and the electrode area was $1.0 \mathrm{~cm}^{2}$. (B) Integrated current from all cycles vs cycle number. (C) Extinction in dark state vs integrated current with linear fits. (D) Contrast as $\Delta T$ for different thicknesses (cycle numbers) of the PProDOTMe ${ }_{2}$ film, identifying the optimal thicknesses for different wavelengths representing RGB and also the wavelength of maximal contrast $(580 \mathrm{~nm})$. The contribution from the extinction of the thin Au film has been removed.

no nanoholes on the red samples (and thus no scattering) because they did not improve the color quality. This is because the plasmonic activity occurs in the red region, which was further confirmed by dispersion relation calculations (Figure S1). The chromaticity coordinates in the 1931 CIE 2D diagram are shown in Figure 1C, together with the standard RGB gamut. The metasurfaces have a reasonable gamut, similar to other plasmonic structures, ${ }^{1}$ and it is well-oriented around the D65 white point $(0.31,0.33)$. Figure $1 \mathrm{D}$ shows an example of micropatterned metasurfaces (RGB stripes). The complementary colors seen under dark-field illumination represent the coloration contribution by resonant excitation of surface plasmons. ${ }^{3,27}$ In summary, these metasurfaces have similar reflectivity and chromaticity as in previous work, but with the benefit of stability in nonaqueous electrolytes.

It is important to note that structural colors that rely on ambient light never provide both excellent chromaticity and reflectivity: If a surface would reflect a very narrow resonant wavelength band $(\sim 10 \mathrm{~nm})$, the color quality would improve, but the visibility would be extremely poor because only a small fraction of the illumination light is reflected. ${ }^{10}$ Therefore, a compromise between chromaticity and brightness is always necessary. In an emissive display (e.g., LED), the situation is different because the narrow emission bands can become clearly visible by increasing intensity (and power consumption). The metasurfaces offer a good balance between chromaticity and reflectivity with their line widths of $\sim 100$ $\mathrm{nm}$, representing a third of the visible region for each primary color.

In order to switch the colors on and off in an optimal manner, we evaluated several conductive polymers prepared by electropolymerization, as well as tungsten oxide $\left(\mathrm{WO}_{3}\right)$ films prepared by reactive sputtering. ${ }^{32}$ Starting with the polymers, we have previously tested polypyrrole ${ }^{3}$ (PPy) and poly $(3,4-$ ethylenedioxythiophene $)^{27}$ (PEDOT), but literature suggests that dimethylpropylene-dioxythiophene (PProDOTMe $)_{2}$ gives better contrast, based on transmission mode measurements on transparent conductors. ${ }^{21}$ However, there appears to be a lack of reports on electropolymerization of PProDOTMe $\mathrm{PP}_{2}$ on thin gold films prepared by standard vacuum deposition. Such gold layers are frequently used in plasmonics for their long-term stability (especially in electrochemistry) and provide good structural colors (e.g., Figure 1). The work by the group of Reynolds has used porous gold structures in special cell configurations $^{33,34}$ or gold films formed by electroless precipitation. $^{35}$ For plasmonic structures, the polymer has only been implemented on aluminum. ${ }^{12}$ Using previous results as a starting point, we evaluated different recipes for electropolymerization of PProDOTMe 2 on thin planar gold layers and found that the most homogeneous and reproducible films were achieved by voltage sweeps performed repeatedly. For comparison, maintaining a constant potential or current resulted in films that were not uniform and the process had poor reproducibility (Figure S2). Figure 2A shows cyclic 

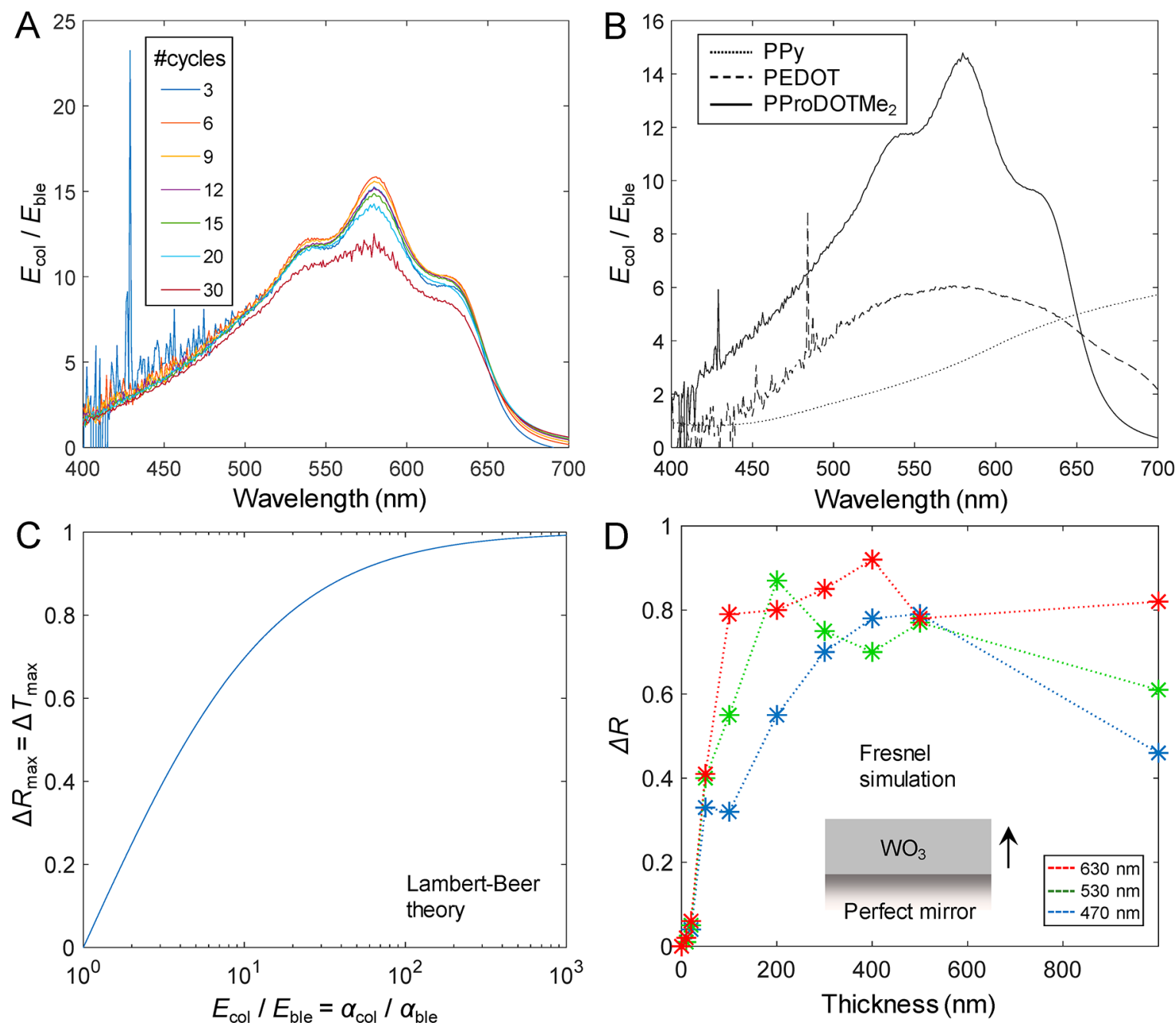

Figure 3. (A) Extinction ratio for different polymer thickness. The contribution from the Au support and the liquid cell was subtracted from the extinction values. (B) Extinction ratio for different electrochromic polymers. (C) Theoretical maximum contrast for a perfect mirror $\left(R_{0}=1\right)$ as a function of extinction ratio (essentially a plot of eq 4). (D) Results of Fresnel models to determine contrast of $\mathrm{WO}_{3}$ films of different thickness in reflection mode (normal incidence) for a perfect mirror support.

voltammetry results of the electropolymerization, which starts at about $+0.8 \mathrm{~V}\left(\mathrm{vs} \mathrm{Ag} / \mathrm{Ag}^{+}\right)$. The voltage range was from -1.0 to $+1.1 \mathrm{~V}$ in order to ensure the formed polymer stayed to the surface. In subsequent cycles the oxidation and reduction peaks of the polymer film start to become visible. The scan rate was set as a compromise between precision and uniformity: faster sweeps give less polymer deposition per cycle and better precision in film growth, but less uniform coatings were observed. At $200 \mathrm{mV} / \mathrm{s}$, the films were homogeneous over the whole area of $1 \mathrm{~cm}^{2}$ by visual inspection. The integrated current (charge) from all voltage sweeps scaled almost linearly with cycle number (Figure 2B), showing that the same amount of polymer is added in each step. However, there was some variation in the charge transfer per cycle in different experiments, which means that it is important to record the current to know the end thickness. We also noted that intense light, such as the beam used to measure the spectrum, could influence the polymerization process. (Hence the light was off during polymerization.) The charge transfer was approximately linear with the dry thickness according to profilometer measurements, and $1 \mathrm{mC} / \mathrm{cm}^{2}$ corresponded to a dry thickness of $\sim 10 \mathrm{~nm}$ (Figure S3). Assuming a density of $\sim 1 \mathrm{~g} / \mathrm{cm}^{3}$, the electron transfer per monomer grown is 1.9 (monomer weight
$184 \mathrm{~g} / \mathrm{mol}$ ), which is close to the expected number of two electrons per bond formed.

We also measured the extinction of the PProDOTMe films in the dark state $(-1 \mathrm{~V})$ as a function of charge transfer, showing a perfectly linear relation (Figure $2 \mathrm{C}$ ), which verifies that the Lambert-Beer law (eq 1) is applicable. At voltages below $-1 \mathrm{~V}$ or above approximately $+0.5 \mathrm{~V}$ the change in transmission was negligible $(<1 \%)$ for the polymer films. Upon switching the polymer ( $1 \mathrm{M} \mathrm{LiClO}_{4}$ in PC) between transparent and absorbing (Figure 2D), the contrast was found to depend on film thickness, and an optimum for transmission mode could be identified, in agreement with eq 3 . Note that the specific thickness that gives optimal contrast, is wavelength-dependent and does not necessarily give the highest optical contrast on a nanostructured surface that has plasmonic activity, because then the plasmons are also switched on and off. ${ }^{3}$ However, it serves as a good first approximation to assume that the thickness which gives $\Delta R_{\max }$ should be about half the value identified for transmission mode. The maximum contrast of $77 \%$ at $580 \mathrm{~nm}$ is in excellent agreement with previous reports for PProDOTMe $\mathrm{P}_{2}$ prepared on transparent conductors, ${ }^{21}$ which shows that our electropolymerization procedure for gold works satisfactory. 

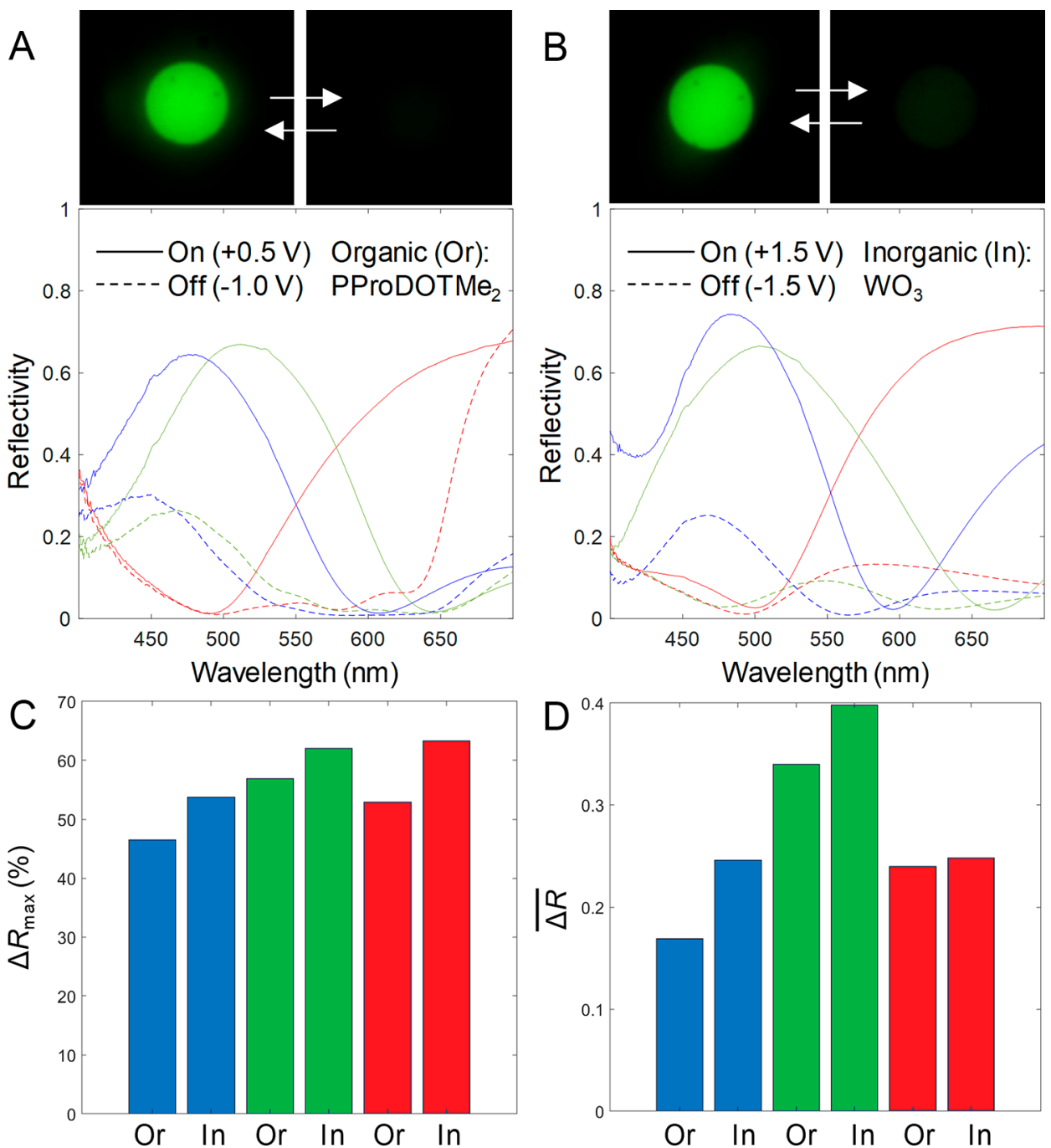

Figure 4. Optimized contrast for switching of metasurfaces with primary colors. (A) Reflectivity spectra in bright and dark states for metasurfaces

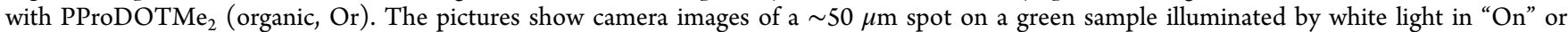
"Off" states (same camera settings). (B) Same as (A) but for metasurfaces with $\mathrm{WO}_{3}$ (inorganic, In). (C) Contrast comparison for each electrochromic metasurface in terms of highest change in absolute reflectivity (at whichever wavelength gives $\Delta R_{\max }$ ). (D) Similar contrast comparison for each metasurface with the luminosity function as weight (eq 6).

To evaluate the contrast, we plotted the extinction ratio for the on and off states for different polymer thickness, which should remain constant according to eq 5 . In order to acquire only the film properties, we subtracted the background extinction (mainly reflection) from the thin gold film and the flow cell. Indeed, the extinction ratio showed little variation (Figure 3A) and no trend of increasing or decreasing with thickness. To compare the different polymers, we plotted their averaged extinction ratios together (Figure 3B). From this comparison it is clear that, as suspected, ${ }^{21}$ PProDOTMe $_{2}$ provides the highest extinction ratio over almost the whole visible. (However, the other polymers were much easier to synthesize as they were insensitive to how the voltage was applied during electropolymerization.) Figure 3C shows a semilog plot based on eq 4 to visualize how high extinction ratios that need to be achieved to generate a certain contrast in theory. One well-established conductive polymer not included in this comparison is polyaniline, ${ }^{5,12,15}$ but it is generally not considered as a candidate for black/transparent switching.

Importantly, the switching properties of the inorganic $\mathrm{WO}_{3}$ films (characterization in Figure S4) could not be evaluated in the same manner due to thin film interference as the real part of the refractive index was around 2.2 (Figure S5). This differs from the organic films, which have a refractive index $(n \approx 1.5$ for polymers $)$ similar to that of the electrolyte $(n=1.42$ for PC), which effectively removes one optical boundary. Full Fresnel models had to be used to evaluate the contrast, using literature values of the permittivity of $\mathrm{WO}_{3}$ in bright and dark states $^{30}$ (keeping in mind that the exact values may depend on how the $\mathrm{WO}_{3}$ is prepared). Figure 3D shows an example of calculated contrast in reflection mode by simulating a $\mathrm{WO}_{3}$ film with variable thickness on a perfect mirror support for red, green, and blue (470,530, and $630 \mathrm{~nm}$, respectively). The simulated metal oxide film did not give the same smooth curves with a single maximum at a certain thickness, as observed for the experimental polymer characterization $(\Delta T$ in Figure $2 \mathrm{D})$. Still, $\Delta R$ can clearly reach values considerably higher $(>80 \%)$ than for all the polymers given that the thin film interference acts in favor of a large contrast at the wavelength of interest. Therefore, the $\mathrm{WO}_{3}$ films may outperform PProDOTMe $\mathrm{M}_{2}$, although implementation on the metasurfaces requires more careful optimization. 
Next, we present the highest reflectivity changes we could achieve for our metasurfaces with organic (Figure 4A) or inorganic (Figure 4B) electrochromism. When implementing $\mathrm{PProDOTMe}_{2}$ on the metasurfaces, we electropolymerized a film close to the optimal value determined for each color (Figure 2D) and then continued to polymerize in steps (voltage sweeps) while monitoring the contrast when switching. Due to contribution from the resonant reflectivity of the metasurfaces, the ideal values of thickness ( 6 cycles for red, 9 for green and 15 for blue) were not identical but quite similar to those identified in transmission mode (Figure 2D) divided by two. The $\mathrm{WO}_{3}$ thickness that gives optimal contrast on a given metasurface was instead selected based on Fresnel models excluding the nanoholes (Figures S6 and S7). Although such calculations do not include the plasmonic contribution to the coloration for blue and green samples, they enabled us to predict which thickness of $\mathrm{WO}_{3}$ would make the thin film interference effects of all layers (four in total) favorable. This gave a $\mathrm{WO}_{3}$ thickness of 130,180 , and $130 \mathrm{~nm}$ for $\mathrm{R}, \mathrm{G}$, and $\mathrm{B}$, respectively. Thus, for both the organic and the inorganic samples, it is possible to avoid a pure trial and error approach when implementing electrochromism: The polymers should be precharacterized in the transmission mode and for the metal oxides the complex permittivity in both coloration states is needed. The end results show that the inorganic devices give the highest contrast for all colors. It did not matter if the contrast was evaluated as the highest $\Delta R$ at any wavelength (Figure $4 C$ ) or the $\Delta R$ weighted by the luminosity function (Figure 4D). Furthermore, the contrast of the inorganic film is indeed very high for all colors $(\Delta R \approx 50 \%)$, and so is the absolute reflectivity in the bright state (up to $\sim 70 \%$ ). Still, the organic films are not far off in performance $(\Delta R \approx 50 \%$, reflectivity $>60 \%$ ), and they are much easier to prepare, that is, electropolymerization as compared to reactive sputtering (with vacuum chamber and plasma). For comparison, in previous work with other materials and aqueous electrolytes, ${ }^{3,27}$ the contrast was considerably lower, especially in the blue region ( 25\%).

Another important parameter for electrochromic devices is the switching speed. We performed a comparison between $\mathrm{PProDOTMe}_{2}$ (Figure 5A) and $\mathrm{WO}_{3}$ (Figure 5B) when switching by potential steps. The intensity of transmitted light at different wavelengths is monitored with high temporal resolution down to a few ms. ${ }^{31}$ Here we simply picked a film thickness $(200 \mathrm{~nm})$ for polymer and metal oxide in the range where the highest contrasts were achieved. (A more detailed analysis of switching speed for different thickness and in different electrochemical cells is the subject of future work.) Figure 5A shows that the organic film is switching reasonably fast $(<1 \mathrm{~s})$ in both directions, and the speed is approximately the same for all wavelengths in the visible. The switch speed was not faster for PEDOT or PPy (not shown). The inorganic $\mathrm{WO}_{3}$ is switching much slower $(\sim 10 \mathrm{~s})$ from the bleached to the colored state, which can be attributed to the slow transport of $\mathrm{Li}^{+}$ions through the film. ${ }^{36}$ Electron microscopy imaging indicated that the polymer films are much more permeable to an electrolyte (Figure S8). The reverse switch (removal of the intercalated $\mathrm{Li}^{+}$) occurs at a speed comparable to that of the organic film, as expected. ${ }^{25}$ As Supporting Information, we provide five different videos showing real-time switching of metasurfaces. (Detailed descriptions of the videos are also in the Supporting Information.) It should be kept in mind that the illumination is white and constant in all these videos.
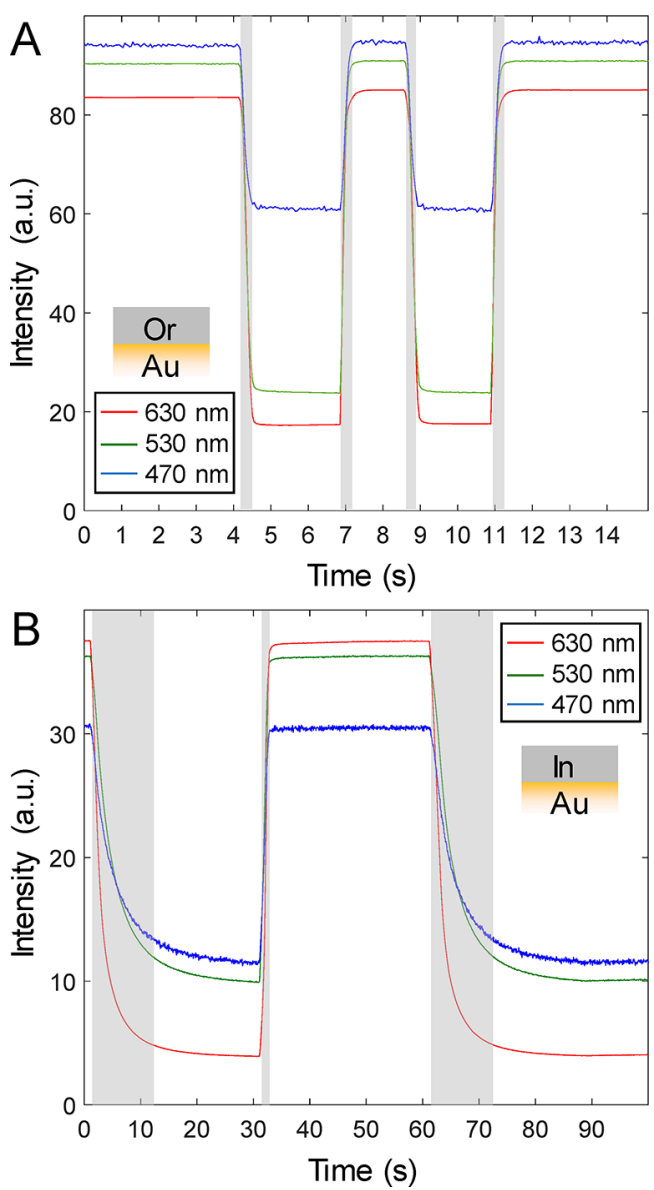

Figure 5. Comparing switching speed by intensity time traces at different wavelengths (square wave voltammetry). (A) Example of time trace when switching PProDOTMe $(\mathrm{Or})$ on gold in $1 \mathrm{M}$ $\mathrm{LiClO}_{4}$ in PC. The time it takes to reach $90 \%$ of the full intensity change is $\sim 600 \mathrm{~ms}$ for dark to bright and $\sim 800 \mathrm{~ms}$ for bright to dark. All wavelengths give approximately the same switching speed $( \pm 100$ $\mathrm{ms}$ ). (B) Example of $\mathrm{WO}_{3}$ (In) switching in the same electrolyte. The time for reaching $90 \%$ of the total switch is marked in gray. The thickness of the PProDOTMe ${ }_{2}$ and the $\mathrm{WO}_{3}$ were both $\sim 200 \mathrm{~nm}$.

The main motivation for electronic paper technologies is power savings by many orders of magnitude as compared to emissive displays, ${ }^{37}$ especially under bright conditions where emissive displays must increase their brightness to overcome ambient light. Unless the device is intended to switch very frequently, the most important parameter to evaluate the power consumption is bistability, that is, the time that a coloration state can be maintained at open circuit voltage. With full bistability, the power consumption is zero for a static state in a display device. We investigated how the intensity changed over time after disconnecting the voltage in the dark state for PProDOTMe 2 (Figure 6A) and $\mathrm{WO}_{3}$ (Figure 6B). The intensity drifts back very slowly in both cases, although $\mathrm{WO}_{3}$ shows slightly better performance. For the polymer, some wavelength dependence can be seen, while the inorganic film shows negligible changes for all colors over at least an hour. In the bright state, the bistability was essentially perfect for both devices since this is close to the equilibrium state (no intercalation in $\mathrm{WO}_{3}$, anions in PProDOTMe $)_{2}$. As an alternative measure of power consumption, the energy per area for one switch can be used. ${ }^{3}$ This gave values of 1-2 mJ/ $\mathrm{cm}^{2}$ for PProDOTMe 2 , and up to $40 \mathrm{~mJ} / \mathrm{cm}^{2}$ for $\mathrm{WO}_{3}$, that is, 

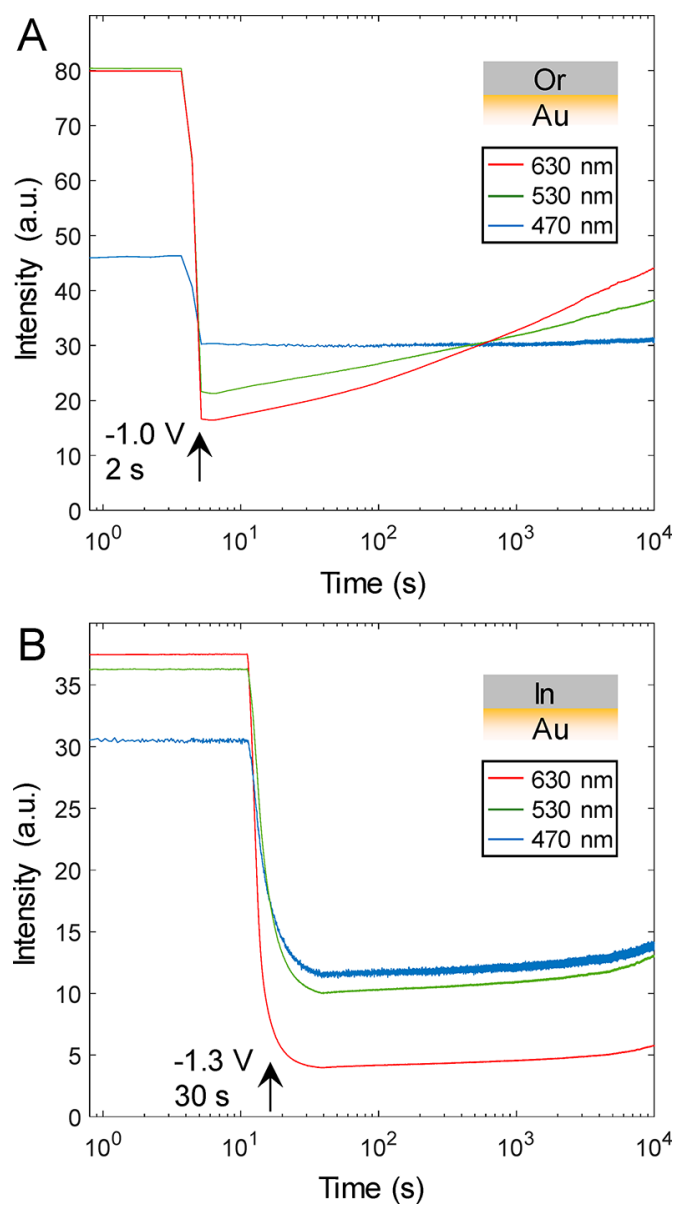

Figure 6. Bistability tests of organic (Or, A) and inorganic (In, B) electrochromic films on Au surfaces. The voltage is switched off after establishing the dark state by a negative pulse and the intensity is monitored over hours (note the logarithmic $x$-axes).

a noticeable difference that becomes important if the devices should be switched frequently.

Finally, we will show that with optimal electrochromic contrast, our metasurfaces can provide much better performance as RGB pixels in a reflective color display than existing technologies. In order to provide colors, electrophoretic displays and LCDs introduce color filters to generate RGB subpixels (Figure 7A). Such displays do exist, but they are generally not found on the market, which arguably is due to their poor image quality in terms of brightness and contrast. ${ }^{10}$ For instance, E-ink markets the Triton, yet such e-reader devices are not found among suppliers (at the time of writing). ${ }^{10}$ The metasurfaces are already red, green, or blue, as prepared, and their reflectivity is high as well as independent of polarization. In an LCD, 50\% of the incident light is lost directly due to the polarizers, so we consider electrophoretic displays to be the most competitive option. (Yet, it should be noted that LCDs have other advantages, such as video speed.) We measured the reflectivity from a (black and white) Amazon Kindle device in the bright and dark states (Figure 7B), using the same instrument as for the metasurfaces. We then assumed that the color filters on RGB pixels would transmit $90 \%$ of each primary color (a typical value for band-pass filters). Hence, the reflectivity from a subpixel of any color in the electrophoretic display is given by the spectra in Figure $7 \mathrm{~B}$ multiplied by 0.81 . In Figure $7 \mathrm{C}$, these values are compared with those of the metasurfaces. Notably, both brightness and contrast are almost twice as high for the metasurfaces, even when using the organic electrochromic films. Thus, it seems plausible that a reflective color display with RGB pixels containing the metasurfaces will have sufficient brightness and contrast to be of practical use in many scenarios. We emphasize, however, that the image quality (also including chromaticity) in such a display will never be as good as for emissive displays. The point is that the plasmonic electronic paper can operate with extremely low power consumption (Figure 6). At the same time, the image quality will be good enough for many applications, especially image display in bright environments.

\section{CONCLUSIONS}

In conclusion, we have shown primary colored metasurfaces with high reflectivity that are electrochemically stable in a nonaqueous electrolyte. This opens new possibilities for implementation of both organic and inorganic electrochromic materials in order to switch the colors on and off. We present a comparison of PProDOTMe , which seems to be the best among established polymers for high-contrast broadband switching, and the well-studied transition metal oxide $\mathrm{WO}_{3}$. We also compare both types of metasurfaces with electrophoretic displays (see Table 1). The inorganic device gives the highest contrast of over $60 \%$ for most wavelengths, but the polymer is not far behind in performance. Using the right methodology, the polymer films are easily synthesized by electropolymerization, while $\mathrm{WO}_{3}$ films require reactive sputtering. The polymer also switches faster $(<1 \mathrm{~s})$, but $\mathrm{WO}_{3}$ has essentially perfect bistability in the electrolyte used. If the optimized electrochromic metasurfaces are implemented in reflective color displays as RGB pixels they will provide superior image quality compared to subpixel generation by color filters. The power consumption of such a display will be extremely low or basically zero when showing a static image.

As a final point, it should be noted that there are a few reports of new types of organic ${ }^{39}$ and inorganic ${ }^{40}$ electrochromic films that claim extremely high contrast when switching between black and transparent states. Our study has focused on the well-established polymer PProdDOTMe and transition metal oxide $\mathrm{WO}_{3}$, both of which have been successfully implemented in electrochromic devices by many research groups worldwide. We believe it remains to be seen if the newer materials truly can outperform the established ones and the methodology presented in this work helps to perform the comparison.

\section{EXPERIMENTAL SECTION}

Chemicals. Propylene carbonate (PC, purity 99\%) was from Sigma-Aldrich. $\mathrm{LiClO}_{4}$ (anhydrous) was from Fisher Scientific. ProDOTMe $\mathrm{M}_{2}$ was purchased from Sycon Polymers India Pvt. Ltd. and purified by making a dispersion in deionized water $(\sim 1 \mathrm{~g}$ monomer in $20 \mathrm{~mL}$ water $)$ by sonication at $40{ }^{\circ} \mathrm{C}$ until a milky liquid was produced together with a brownish liquid. The milky liquid was transferred to another beaker, extracted, and recrystallized with hexane. Pyrrole and EDOT were purchased from Sigma and used without any further purification.

Fabrication of Metasurfaces. $\mathrm{Al}$ (with $5 \mathrm{~nm} \mathrm{Cr}$ adhesion layer), $\mathrm{Al}_{2} \mathrm{O}_{3}$, and $\mathrm{Au}$ (with $1 \mathrm{~nm} \mathrm{Ti}$ adhesion layer) were deposited by evaporation (Lesker PVD 225) onto glass substrates. Prior to $\mathrm{Au}$ deposition, colloidal lithography was 

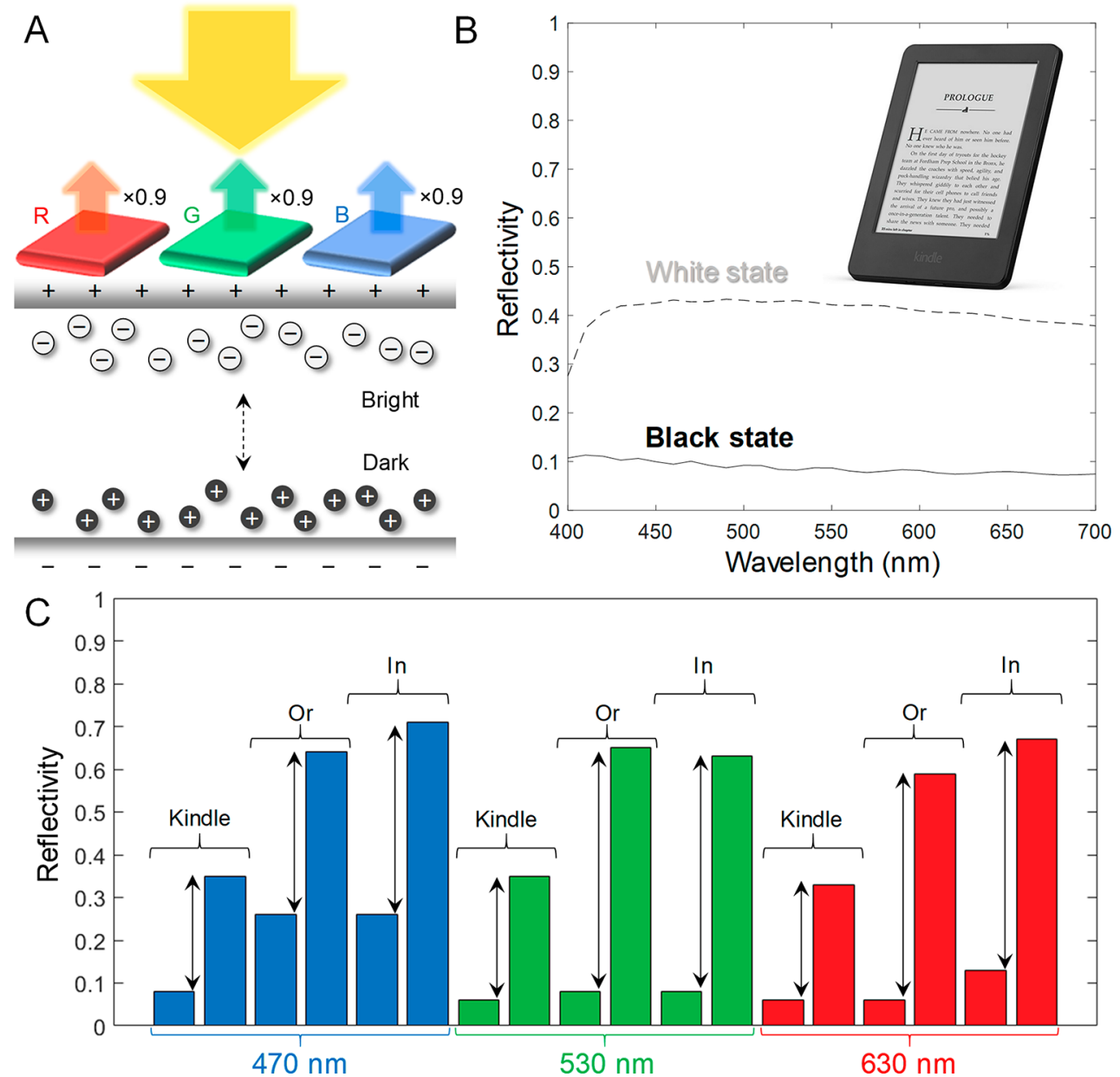

Figure 7. Comparison between metasurfaces and electrophoretic displays for electronic paper in color. (A) Principle of color generation by subpixels containing color filters, assuming $90 \%$ transmission of each primary color. (B) Diffuse reflectivity of black and white states of an Amazon Kindle device, measured with the same instrument as used for the metasurfaces. (C) Reflectivity in on and off states for three wavelengths, comparing the electrophoretic display with the metasurfaces having either organic (Or) or inorganic (In) films.

\section{Table 1}

\begin{tabular}{|c|c|c|c|}
\hline & metasurfaces with PProDOTMe ${ }_{2}$ & metasurfaces with $\mathrm{WO}_{3}$ & electrophoretic \\
\hline preparation & $\begin{array}{l}\text { electropolymerization directly on the } \\
\text { surface }\end{array}$ & requires deposition by reactive sputtering & industrial production \\
\hline brightness (reflectivity) & high $(60-70 \%)$ & high $(60-70 \%)$ & $\begin{array}{l}40 \% \text { in black/white, } \\
35 \% \text { for RGB } \\
\text { subpixels }\end{array}$ \\
\hline contrast & high $(\sim 50 \%)$ & higher $(\sim 60 \%)$ & $\begin{array}{l}30 \% \text { in black/white, } \\
25 \% \text { for RGB } \\
\text { subpixels }\end{array}$ \\
\hline optical modeling & Lambert-Beer formalism & full Fresnel models required & $\begin{array}{l}\text { particle scattering/ } \\
\text { absorption }\end{array}$ \\
\hline switching speed & $0.1-1 \mathrm{~s}$ & $1-10 \mathrm{~s}$, depends on switch direction & $\sim 1 \mathrm{~s}$ \\
\hline bistability & good overall, wavelength dependent & excellent (hours) & excellent \\
\hline $\begin{array}{l}\text { device stability (not } \\
\text { systematically evaluated in } \\
\text { this work) }\end{array}$ & $\begin{array}{l}\text { excellent in terms of number of } \\
\text { cycles } 34\left(\sim 10^{5}\right) \text {; sensitive to intense } \\
\text { light }\end{array}$ & $\begin{array}{l}\text { may need to be regenerated after a certain number of cycles } \\
\left(\sim 10^{2}\right) \text { in order to not lose performance; sensitive to water }\end{array}$ & $\begin{array}{l}\text { commercially viable in } \\
\text { black/white }\end{array}$ \\
\hline
\end{tabular}

performed on $\mathrm{Al}_{2} \mathrm{O}_{3}$, as described previously, ${ }^{3,27}$ using $147 \mathrm{~nm}$ polystyrene-sulfate particles (microParticles $\mathrm{GmbH}$ ). For red samples the colloidal lithography step was omitted. Micropatterns were created by laser lithography, as described previously. ${ }^{3,27}$

Electropolymerization. For initial tests and for transmission mode measurements, substrates consisted of glass with
$20 \mathrm{~nm}$ gold film $(1 \mathrm{~nm} \mathrm{Cr})$ deposited in the same way as for the metasurfaces. The gold surface was first washed with acetone and isopropyl alcohol and dried with nitrogen. The substrate was placed in a custom-made spectro-electrochemical cell (Redox Me $\mathrm{AB}$ ) with a Pt coil as counter electrode and an $\mathrm{Ag}$ electrode in contact with $0.01 \mathrm{M} \mathrm{AgNO}_{3}$ and $0.1 \mathrm{M}$ tetrabutyl ammonium perchlorate as reference $\left(\mathrm{Ag} / \mathrm{Ag}^{+}\right)$. All 
polymers were electrodeposited with a potentiostat (Gamry Interface $1010 \mathrm{E}$ ) from a $10 \mathrm{mM}$ monomer solution in $0.1 \mathrm{M}$ $\mathrm{LiClO}_{4} / \mathrm{PC}$, degassed by a $\mathrm{N}_{2}$ purge. Between every positive $\mathrm{CV}$ scan, a potential of $-1 \mathrm{~V}$ was applied for $10 \mathrm{~s}$ to remove anions inserted in the polymer film. All voltages mentioned are versus the $\mathrm{Ag} / \mathrm{Ag}^{+}$reference. No $i R$ compensation was used. The dry thickness of the samples was measured with a profilometer (KLA Tencor Alpha-Step D-100).

Sputtering. $\mathrm{WO}_{3}$ films were deposited by reactive $\mathrm{RF}$ sputtering inspired by previous recipes ${ }^{32}$ (Nordiko 2000), with flow rates 32 and $8 \mathrm{sccm}$ of $\mathrm{Ar}$ and $\mathrm{O}_{2}$, respectively, at 20 mTorr and with $150 \mathrm{~W}$ power. The sample was kept as close as possible to the source at approximately $65 \mathrm{~mm}$ distance. The thicknesses and permittivity was analyzed using a spectroscopic ellipsometer (J.A.Wollam M2000). A dispersive model with three superimposed oscillators was assumed. XPS characterization was performed with a VersaProbe III (Physical Electronics).

Opto-Electrical Measurements. The diffuse reflectivity spectra of the metasurfaces (without electrochromic films) and of the Kindle device (Paperwhite 2 DP75SDI) were measured with a CM-700d spectrophotometer (Konika Minolta). This instrument also gives the CIE coordinates. Measurements of electrochromic surfaces during switching were performed in a home-built microscopy/reflection setup into which the electrochemical cell was placed and the potentiostat connected. PProDOTMe $\mathrm{M}_{2}$ was switched between $-1.0 \mathrm{~V}$ and up to $+0.8 \mathrm{~V}$ versus $\mathrm{Ag} / \mathrm{Ag}^{+}$for the thickest films. PPy was switched between $-1.0 \mathrm{~V}$ and $+0.4 \mathrm{~V}$ versus $\mathrm{Ag} / \mathrm{Ag}^{+}$, while PEDOT was switched between $-1.0 \mathrm{~V}$ and $+0.4 \mathrm{~V}$ versus $\mathrm{Ag} / \mathrm{Ag}^{+}$. $\mathrm{WO}_{3}$ was switched between $-1.5 \mathrm{~V}$ and $+1.7 \mathrm{~V}$ versus $\mathrm{Ag} / \mathrm{Ag}^{+}$for thin $\mathrm{Au}$ films and $-1.5 \mathrm{~V}$ and $+1.5 \mathrm{~V}$ versus $\mathrm{Ag} / \mathrm{Ag}^{+}$for metasurfaces. All switching was performed in PC with $1 \mathrm{M}$ $\mathrm{LiClO}_{4}$. A $4 \times$ air objective with NA 0.1 illuminated and collected light, representing an angular illumination range up to $4^{\circ}$ to the surface normal for surfaces inside the electrolyte. A tungsten lamp (Azpect Photonics) was used and the spectrometer was a fiber-coupled photodiode array (B\&WTek). A broadband dielectric mirror (BB05-E02, Thorlabs) was used to measure the reference reflection (no cell). It was verified that this mirror had essentially $100 \%$ reflectivity (>99\%) using the Konika Minolta instrument. To account for light refraction, and so on, leading to an intensity loss in the liquid cell due to optics designed for measurements in air, the reflectivity spectrum of an Ag mirror was compared in air and inside the cell with electrolyte. All spectra were then corrected with a factor representing the ratio of these intensities $(\sim 10 \%$ change $)$. For transmission mode measurements, the reference intensity was simply measured without the liquid cell in place. The dark counts in the spectrometer (light source off) were always subtracted in all acquisitions. Note that extinction is defined using the natural logarithm and that it is additive. The contribution from the thin $\mathrm{Au}$ film and the flow cell could thus be removed by subtracting the corresponding extinction spectrum (before depositing an electrochromic film). The luminosity function was obtained from the Commission Internationale de l'Éclairage (CIE).

Pictures and Videos. RGB stripes were imaged using an Axiocam506 color camera in a microscope (Zeiss Axio Observer 7) with a $50 \times$ air objective designed for dark field illumination in reflection mode. Videos of switching of stripes and illuminated spots were recorded with a Thorlabs
DCC1645C CMOS camera. Other photos and videos were obtained with mobile phones.

\section{ASSOCIATED CONTENT}

\section{Supporting Information}

The Supporting Information is available free of charge at https://pubs.acs.org/doi/10.1021/acsphotonics.0c00394.

Video descriptions and Figures S1-S8 (PDF)

Five supporting videos (AVI)

\section{AUTHOR INFORMATION}

\section{Corresponding Author}

Andreas Dahlin - Department of Chemistry and Chemical Engineering, Chalmers University of Technology, 41296 Gothenburg, Sweden; 이이.0rg/0000-0003-1545-5860; Email: adahlin@chalmers.se

\section{Authors}

Marika Gugole - Department of Chemistry and Chemical Engineering, Chalmers University of Technology, 41296 Gothenburg, Sweden

Oliver Olsson - Department of Chemistry and Chemical Engineering, Chalmers University of Technology, 41296 Gothenburg, Sweden

Kunli Xiong - Department of Chemistry and Chemical Engineering, Chalmers University of Technology, 41296 Gothenburg, Sweden

Jolie C. Blake - Department of Chemistry and Chemical Engineering, Chalmers University of Technology, 41296 Gothenburg, Sweden

José Montero Amenedo - Department of Materials Science and Engineering, Uppsala University, SE-75121 Uppsala, Sweden

Ilknur Bayrak Pehlivan - Department of Materials Science and Engineering, Uppsala University, SE-75121 Uppsala, Sweden; ( $)$ orcid.org/0000-0002-4362-6148

Gunnar A. Niklasson - Department of Materials Science and Engineering, Uppsala University, SE-75121 Uppsala, Sweden; (1) orcid.org/0000-0002-8279-5163

Complete contact information is available at:

https://pubs.acs.org/10.1021/acsphotonics.0c00394

\section{Author Contributions}

† These authors contributed equally.

\section{Notes}

The authors declare the following competing financial interest(s): Two authors (A.D. and K.X.) are co-founders of the company rdot displays $A B$, which works with reflective display technologies. O.O. has shares in this company. The authors have no active role in the company anymore. The company has not influenced the study in any way.

\section{ACKNOWLEDGMENTS}

We thank Anne Wendel at Chalmers for help with XPS analysis. Assoc. Prof. Magnus Jonsson at Linköping University and Dr. Peter Andersson Ersman at RISE are acknowledged for useful discussions. This work was financed by the Swedish Foundation for Strategic Research (EM16-0002). 


\section{REFERENCES}

(1) Kristensen, A.; Yang, J. K. W.; Bozhevolnyi, S. I.; Link, S.; Nordlander, P.; Halas, N. J.; Mortensen, N. A. Plasmonic colour generation. Nat. Rev. Mater. 2017, 2, 16088.

(2) Freestone, I.; Meeks, N.; Sax, M.; Higgitt, C. The Lycurgus Cup A Roman nanotechnology. Gold Bull. 2007, 40, 270-277.

(3) Xiong, K.; Emilsson, G.; Maziz, A.; Yang, X.; Shao, L.; Jager, E. W. H.; Dahlin, A. B. Plasmonic metasurfaces with conjugated polymers for flexible electronic paper in color. Adv. Mater. 2016, 28, 9956-9960.

(4) Li, Y.; van de Groep, J.; Talin, A. A.; Brongersma, M. L. Dynamic tuning of gap plasmon resonances using a solid-state electrochromic device. Nano Lett. 2019, 19, 7988-7995.

(5) Shahabuddin, M.; McDowell, T.; Bonner, C. E.; Noginova, N. Enhancement of electrochromic polymer switching in plasmonic nanostructured environment. ACS Appl. Nano Mater. 2019, 2, 17131719.

(6) Wang, Z.; Wang, X.; Cong, S.; Chen, J.; Sun, H.; Chen, Z.; Song, G.; Geng, F.; Chen, Q.; Zhao, Z. Towards full-colour tunability of inorganic electrochromic devices using ultracompact fabry-perot nanocavities. Nat. Commun. 2020, 11, 302.

(7) Hopmann, E.; Elezzabi, A. Y. Plasmochromic nanocavity dynamic light color switching. Nano Lett. 2020, 20, 1876-1882.

(8) Atighilorestani, M.; Jiang, H.; Kaminska, B. Electrochromicpolymer-based switchable plasmonic color devices using surface-relief nanostructure pixels. Adv. Opt. Mater. 2018, 6, 1801179.

(9) Shao, L.; Zhuo, X.; Wang, J. Advanced plasmonic materials for dynamic color display. Adv. Mater. 2018, 30, 1704338.

(10) Xiong, K.; Tordera, D.; Jonsson, M. P.; Dahlin, A. B. Active control of plasmonic colors: emerging display technologies. Rep. Prog. Phys. 2019, 82, 024501.

(11) Franklin, D.; Frank, R.; Wu, S.-T.; Chanda, D. Actively addressed single pixel full-colour plasmonic display. Nat. Commun. 2017, 8, 15209.

(12) Xu, T.; Walter, E. C.; Agrawal, A.; Bohn, C.; Velmurugan, J.; Zhu, W.; Lezec, H. J.; Talin, A. A. High-contrast and fast electrochromic switching enabled by plasmonics. Nat. Commun. 2016, 7, 10479.

(13) Atighilorestani, M.; dos Santos, D. P.; Jaimes, R. F. V. V.; Rahman, M. M.; Temperini, M. L. A.; Brolo, A. G. Electrochemical control of light transmission through nanohole electrode arrays. ACS Photonics 2016, 3, 2375-2382.

(14) Jiang, N. N.; Shao, L.; Wang, J. F. (Gold nanorod core)/ (polyaniline shell) plasmonic switches with large plasmon shifts and modulation depths. Adv. Mater. 2014, 26, 3282-3289.

(15) Leroux, Y. R.; Lacroix, J. C.; Chane-Ching, K. I.; Fave, C.; Felidj, N.; Levi, G.; Aubard, J.; Krenn, J. R.; Hohenau, A. Conducting polymer electrochemical switching as an easy means for designing active plasmonic devices. J. Am. Chem. Soc. 2005, 127, 16022-16023.

(16) Deb, S. K. In Some aspects of electrochromic display systems, Proc. 24th Electronic Components Conf., IEEE, 1974; pp 11-14.

(17) Green, S.; Backholm, J.; Georen, P.; Granqvist, C. G.; Niklasson, G. A. Electrochromism in nickel oxide and tungsten oxide thin films: Ion intercalation from different electrolytes. Sol. Energy Mater. Sol. Cells 2009, 93, 2050-2055.

(18) Granqvist, C. G. Electrochromics for smart windows: Oxidebased thin films and devices. Thin Solid Films 2014, 564, 1-38.

(19) Malti, A.; Brooke, R.; Liu, X.; Zhao, D.; Andersson Ersman, P.; Fahlman, M.; Jonsson, M. P.; Berggren, M.; Crispin, X. Freestanding electrochromic paper. J. Mater. Chem. C 2016, 4, 9680-9686.

(20) Padilla, J.; Osterholm, A. M.; Dyer, A. L.; Reynolds, J. R. Process controlled performance for soluble electrochromic polymers. Sol. Energy Mater. Sol. Cells 2015, 140, 54-60.

(21) Welsh, D. M.; Kumar, A.; Meijer, E. W.; Reynolds, J. R. Enhanced contrast ratios and rapid switching in electrochromics based on poly(3,4-propylenedioxythiophene) derivatives. Adv. Mater. 1999, 11, 1379-1382.
(22) Padilla, J.; Seshadri, V.; Sotzing, G. A.; Otero, T. F. Maximum contrast from an electrochromic material. Electrochem. Commun. 2007, 9, 1931-1935.

(23) Heikenfeld, J.; Drzaic, P.; Yeo, J. S.; Koch, T. A critical review of the present and future prospects for electronic paper. J. Soc. Inf. Disp. 2011, 19, 129-156.

(24) Hayes, R. A.; Feenstra, B. J. Video-speed electronic paper based on electrowetting. Nature 2003, 425, 383-385.

(25) Xu, J.; Zhang, Y.; Zhai, T.-T.; Kuang, Z.; Li, J.; Wang, Y.; Gao, Z.; Song, Y.-Y.; Xia, X.-H. Electrochromic-tuned plasmonics for photothermal sterile window. ACS Nano 2018, 12, 6895-6903.

(26) Kawahara, J.; Ersman, P. A.; Engquist, I.; Berggren, M. Improving the color switch contrast in PEDOT:PSS-based electrochromic displays. Org. Electron. 2012, 13, 469-474.

(27) Xiong, K.; Tordera, D.; Emilsson, G.; Olsson, O.; Linderhed, U.; Jonsson, M. P.; Dahlin, A. B. Switchable plasmonic metasurfaces with high chromaticity containing only abundant metals. Nano Lett. 2017, 17, 7033-7039.

(28) Wang, X.; Chen, K.; de Vasconcelos, L. S.; He, J.; Shin, Y. C.; Mei, J.; Zhao, K. Mechanical breathing in organic electrochromics. Nat. Commun. 2020, 11, 211.

(29) Junesch, J.; Sannomiya, T.; Dahlin, A. B. Optical properties of nanohole arrays in metal-dielectric double films prepared by maskon-metal colloidal lithography. ACS Nano 2012, 6, 10405-10415.

(30) Triana, C. A.; Granquist, C. G.; Niklasson, G. A. Electrochromism and small-polaron hopping in oxygen deficient and lithium intercalated amorphous tungsten oxide films. J. Appl. Phys. 2015, 118, 024901.

(31) Dahlin, A. B.; Zahn, R.; Voros, J. Nanoplasmonic sensing of metal-halide complex formation and the electric double layer capacitor. Nanoscale 2012, 4, 2339-2351.

(32) Yamada, Y.; Tabata, K.; Yashima, T. The character of WO3 film prepared with RF sputtering. Sol. Energy Mater. Sol. Cells 2007, 91, 29-37.

(33) Argun, A. A.; Berard, M.; Aubert, P. H.; Reynolds, J. R. Backside electrical contacts for patterned electrochromic devices on porous substrates. Adv. Mater. 2005, 17, 422-426.

(34) Aubert, P.-H.; Argun, A. A.; Cirpan, A.; Tanner, D. B.; Reynolds, J. R. Microporous patterned electrodes for color-matched electrochromic polymer displays. Chem. Mater. 2004, 16, 2386-2393.

(35) Argun, A. A.; Reynolds, J. R. Line patterning for flexible and laterally configured electrochromic devices. J. Mater. Chem. 2005, 15, 1793-1800.

(36) Malmgren, S.; Green, S. V.; Niklasson, G. A. Anomalous diffusion of ions in electrochromic tungsten oxide films. Electrochim. Acta 2017, 247, 252-257.

(37) Fernandez, M. R.; Casanova, E. Z.; Alonso, I. G. Review of display technologies focusing on power consumption. Sustainability 2015, 7, 10854-10875.

(38) Wen, R.-T.; Granqvist, C. G.; Niklasson, G. A. Eliminating degradation and uncovering ion-trapping dynamics in electrochromic WO3 thin films. Nat. Mater. 2015, 14, 996.

(39) Zhang, Q.; Tsai, C.-Y.; Li, L.-J.; Liaw, D.-J. Colorless-to-colorful switching electrochromic polyimides with very high contrast ratio. Nat. Commun. 2019, 10, 1239.

(40) Cai, G.; Cui, M.; Kumar, V.; Darmawan, P.; Wang, J.; Wang, X.; Lee-Sie Eh, A.; Qian, K.; Lee, P. S. Ultra-large optical modulation of electrochromic porous WO3 film and the local monitoring of redox activity. Chem. Sci. 2016, 7, 1373-1382. 